\title{
Pentose Phosphate Pathway and Glutathione System of Red Blood Cells at the Exacerbation of Chronic Cytomegalovirus Infection during Pregnancy
}

\author{
Michael T. Lucenko, PhD, ScD*; Irina A. Andrievskaya, PhD, ScD \\ Far Eastern Scientific Center of Physiology and Pathology of Respiration, \\ Siberian Branch of Russian Academy of Sciences \\ Blagoveshchensk, the Russian Federation
}

\begin{abstract}
Objective: To determine the activity of the pentose phosphate pathway (PPP) and glutathione system (GS) in red blood cells (RBCs) of pregnant women at 28 to 30 weeks of gestation during the exacerbation of chronic CMV infection (CMVI).

Methods: The study included 50 pregnant women at 28 to 30 weeks of gestation: 25 CMV-seropositive pregnant women (the main group) with CMVI exacerbation and $25 \mathrm{CMV}$-seronegative pregnant women (the control group). We determined the activity of G6PD, GR, GP, and the amount of reduced glutathione and NADP; fatty acid peroxide levels in RBCs; the amount of degenerative forms of RBCs; RBC deformation ability; and indicators of the oxygenated form of hemoglobin and 2,3-DPG.

Results: In RBCs of pregnant women with CMVI exacerbation, cytophotometry analysis of blood smears showed a 2.16-times reduction in the intensity of histochemical reaction to G6P, a 3.3-times reduction in the intensity of histochemical reaction to reduced glutathione, and a 3.1-times reduction in the intensity of histochemical reaction to reduced NADP, compared with the control group. There were disorders in functioning and structural equivalence in both the disulfide reductase system and oxidative systems of PPP in RBCs, which was reflected in lower activity of GR and GP, 1.9 times and 2.5 times, respectively, in the main group. At the same time, the content of 2,3-DPG was 1.24 times more, whereas RBC deformability was 2.25 times less than in the control group.

Conclusion: CMVI exacerbation at 28 to 30 weeks of gestation causes the inhibition of the metabolic activity of the enzymes of PPP and GS, an initiation of membrane destruction oxidative processes that enhance the deformability of RBCs and decrease oxygen metabolism, which creates the risk of anemia and hemic hypoxia in pregnant women. (Int J Biomed. 2017; 7(1):32-36.)
\end{abstract}

Key Words: cytomegalovirus infection • pregnancy $\bullet$ red blood cells $\bullet$ pentose phosphate pathway $\bullet$ glutathione system

\section{Abbreviations}

CMV, cytomegalovirus; 2,3-DPG, diphosphoglycerate; G6P, glucose-6-phosphate; G6PD, glucose-6-phosphate dehydrogenase; GR, glutathione reductase; GP, glutathione peroxidase; GS, glutathione system; PPP, pentose phosphate pathway; RBCs, red blood cells.

\section{Introduction}

CMV is an extremely common virus that can infect almost any one. During pregnancy, a direct effect of CMV on the structural and functional state of immune and hematopoietic cells is very pronounced.$^{[1]} \mathrm{RBCs}$ are actively involved in

*Corresponding author: Prof. Michael T. Lucenko, Academician of the RAS, Far Eastern Scientific Center of Physiology and Pathology of Respiration of the RAS. Blagoveshchensk, Russia. E-mail:Lucenkomt@,mail.ru the interactions of CMV through glycophorin receptors, ${ }^{[2,3]}$ which lead to the initiation of the hydrolytic and proteolytic processes, altering the redox state and the energy balance of RBCs by inhibiting enzymatic reactions in the initial phase of PPP and GS. Disturbances in the erythrocyte metabolism lead to anemia, which is often diagnosed during pregnancy. ${ }^{[4]}$ However, there are no data on the role of chronic CMVI in the formation of anemia in pregnant women during the third trimester of gestation, a period which is extremely important for fetal development. 
The aim of this study was to determine the peculiarities of PPP function and GS activity in RBCs of pregnant women at 28 to 30 weeks of gestation during the exacerbation of chronic CMV infection (CMVI).

\section{Study Design}

We performed a prospective case-control study. The study was performed at a single institution for one year. The study included 50 pregnant women at 28 to 30 weeks of gestation: $25 \mathrm{CMV}$-seropositive pregnant women (the main group) with CMVI exacerbation and $25 \mathrm{CMV}$-seronegative pregnant women (the control group). The two groups were matched in age ( $24.8 \pm 0.3$ years and $23.9 \pm 0.4$ years). Written informed consent was obtained from all patients.

Inclusion criteria for the main group were a relapse of CMVI with clinical signs of acute respiratory viral infections and herpes virus infection (HHV-1,2) remission during the entire gestation period.

Exclusion criteria were primary CMVI, an aggravation of other inflammatory diseases of extragenital localization and sexually transmitted infections.

The clinical diagnosis of primary CMVI was determined by the presence in the peripheral blood of anti-CMV IgM antibody, low-avidity IgG (avidity index $<65 \%$ ), and the presence of CMV DNA in the samples of blood or urine; the relapse of CMVI was diagnosed by the presence of anti-CMV IgM, high-avidity $\operatorname{IgG}$ (avidity index $>65 \%$ ), and the presence of CMV DNA in samples of buccal epithelium and cervical mucosa.

\section{Description of intervention}

We determined the activity of G6PD, GR, GP, and the amount of reduced glutathione and NADP; fatty acid peroxide levels in RBCs; the amount of degenerative forms of RBCs; $\mathrm{RBC}$ deformation ability; and indicators of the oxygenated form of hemoglobin and 2,3-DPG.

At 28 to 30 weeks of gestation, blood samples were collected from the ulnar vein to perform histochemical reactions, PCR, ELISA and spectrophotometric assays. Samples of urine, buccal epithelium, and cervical content were collected for PCR analysis. All samples of biological materials were examined simultaneously.

Primary endpoints: the activity of G6PD, GR, GP, and the amount of reduced glutathione and NADPH, fatty acid peroxide levels, the amount of degenerative forms of RBCs, and RBC deformation ability.

Secondary endpoints: the indicators of oxygenated form of hemoglobin and 2,3-DPG.

\section{Methods of measuring outcomes}

Blood samples $(5 \mathrm{ml})$ were collected from the ulnar vein in standard vacuum tubes with EDTA to obtain the samples of mononuclear cells. For serological tests, we used blood that does not contain anticoagulants. Mononuclear cell isolation for PCR was carried out with Ficoll-Urografin $(\mathrm{d}-1.077 \mathrm{~g} / \mathrm{ml})$
("DNA-Technology", Russia). Serological studies were performed in paired serum samples at intervals of 10-14 days. The morning urine specimens for PCR analysis were collected in a sterile container $(60 \mathrm{ml})$. Buccal epithelium and contents of the cervical canal were collected in standard sterile plastic tubes $(0.5 \mathrm{ml})$ with a physiological solution.

G6PD activity and an amount of reduced NADP were determined by the cytochemical methods in native erythrocytes. The cytochemical assay is based on the reduction of water-soluble colorless tetranitro blue tetrazolium via the electron carrier 1-methoxyphenazine methosulfate, in its water-insoluble dark-colored formazan by NADPH. Darkpurple granules are present in erythrocytes that contain G6PD activity, whereas G6PD-deficient erythrocytes remain unstained. The same principle has been used for detection of glutathione according to the modified method of MT Lucenko and IA Andriyevskaya. ${ }^{[5]}$

The levels of the fatty acid peroxides were determined according to Winkler-Schulze. For control, RBCs were incubated in a medium containing adequate amounts of the substrate instead of a phosphate buffer. Reactions were performed according to the prescriptions listed in Table 1. The obtained smears were studied with the digital microscope MEJI (Japan) connected to a computer according to the Scion program (USA). The activity of reaction products was calculated automatically in a cytophotometric study and expressed in pixels $/ \mu \mathrm{m}^{2}$.

Table 1.

Composition and quantity of reagents used in histochemical reactions

\begin{tabular}{|c|c|c|}
\hline $\begin{array}{l}\text { Name of } \\
\text { reaction }\end{array}$ & Composition of incubation solution & Amount \\
\hline G6PD & $\begin{array}{l}0.1 \mathrm{M} \text { phosphate buffer, } \mathrm{pH} 7.5 \\
\text { Nitroblue tetrazolium (ICN Biomedicals, } \\
\text { USA) } \\
\text { NADP (Appilichem, Germany) } \\
1 \mathrm{M} \mathrm{Na-G6P(ICN} \mathrm{Biomedicals,} \mathrm{CWA)} \\
\mathrm{MgSO}_{4} \text { (Reachim, Russia) } \\
\end{array}$ & $\begin{array}{c}1 \mathrm{ml} \\
1 \mathrm{mg} \\
2 \mathrm{mg} \\
0.3 \mathrm{ml} \\
2 \mathrm{mg} \\
\end{array}$ \\
\hline NADP & $\begin{array}{l}\text { 0.1 M phosphate buffer, pH } 7.4 \\
\text { NADP (Appilichem, Germany) } \\
\text { Nitroblue tetrazolium (ICN Biomedicals, } \\
\text { CШA) }\end{array}$ & $\begin{array}{l}1 \mathrm{ml} \\
1 \mathrm{ml} \\
1 \mathrm{mg}\end{array}$ \\
\hline Glutathione & $\begin{array}{l}10 \% \text { aqueous solution of sodium } \\
\text { nitroprusside (Biochemreactiv, Russia) } \\
2 \% \text { ammonia solution (Reahim, Russia) }\end{array}$ & $\begin{array}{l}0.25 \mathrm{ml} \\
0.25 \mathrm{ml} \\
\end{array}$ \\
\hline $\begin{array}{l}\text { Fatty } \\
\text { acid peroxides }\end{array}$ & $\begin{array}{l}576 \mathrm{mg} \text { of } \alpha \text {-naphthol (ICN Biomedicals, } \\
\text { USA) in } 6 \mathrm{ml} \text { of } 1 \mathrm{~N} \mathrm{NaOH} \text { (Reachim, } \\
\text { Russia) filled up to } 50 \mathrm{ml} \text { with distilled } \\
\text { water } \\
691 \mathrm{mg} \text { of n-amino-N,N-dimethyl-phenylen- } \\
\text { ediamine (Laverna, Russia) in } 50 \mathrm{ml} \text { of } \\
\text { distilled water } \\
\text { Lugol's Solution of Iodine } 2 \% \\
0.005 \% \text { solution of lithium carbonate (ICN } \\
\text { Biomedicals, USA) }\end{array}$ & $\begin{array}{l}0.25 \mathrm{ml} \\
0.25 \mathrm{ml} \\
0.5 \mathrm{ml} \\
0.5 \mathrm{ml}\end{array}$ \\
\hline
\end{tabular}

RBC shape was determined by cytofluorimetric analysis using the MEKOS device (registration certificate of Ministry of Health of the Russian Federation 29/10010198/1282-01). 
The erythrocyte membrane density was measured using a digital camera according to the BioVision Pixera program (USA) at a constant of 60 pixels, which corresponds to the morphofunctional state of degenerative forms of RBCs. $\mathrm{RBC}$ deformation properties were calculated using the cytophotometric device Mekos according to the method of MT Lucenko and IA Andriyevskaya. ${ }^{[6]}$

Oxyhemoglobin was determined spectrophotometrically by the method of Malloy and Evelyn. ${ }^{[7]}$ 2,3-DPG level was determined by NS Luganov and MN Blinov. ${ }^{[8]}$ The activity of GR and GP was determined spectro-photometrically using standard reagent kits (Sentinel Diagnostics, Italy).

Statistical analysis was performed using StatSoft Statistica v6.0. The mean (M) and standard error of the mean (SEM) were calculated. For data with normal distribution, inter-group comparisons were performed using Student's t-test. Two-tailed $P<0.05$ was considered statistically significant.

\section{Results}

\section{Primary endpoints}

In RBCs of pregnant women with CMVI exacerbation at 28 to 30 weeks of gestation, cytophotometry analysis of blood smears showed a 2.16-times reduction in the intensity of histochemical reaction to G6P $(\mathrm{P}<0.001)$ (Fig.1), a 3.3-times reduction in the intensity of histochemical reaction to reduced glutathione $(\mathrm{P}<0.001)$ (Fig.2), and a 3.1-times reduction in the intensity of histochemical reaction to reduced NADP $(\mathrm{P}<0.001)$ (Fig.3), compared with the control group, indicating suppressed PPP activity in RBCs caused by alteration in the acceptor properties of NADP, in the main coenzyme of dehydrogenases, and in thiol-disulfide reactions of the redox svstem defined bv glutathione.
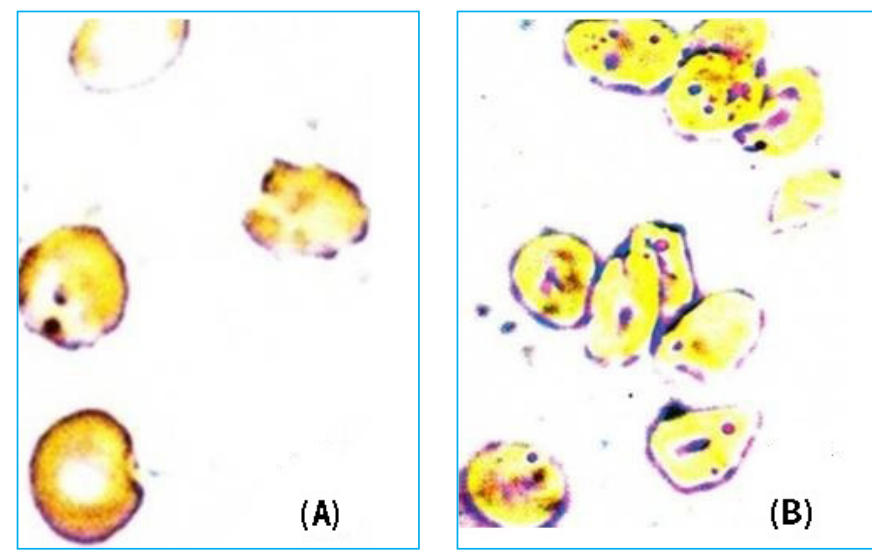

Fig. 1. RBCs of a pregnant woman with CMVI exacerbation at 28 weeks of gestation. Histochemical reaction to G6PD according to $R$. Lilly. (A) - main group, $(B)$ - control group. Magnification: $15 \times 100$.

At the same time, there were disorders in functioning and structural equivalence in both the disulfide reductase system and oxidative systems of PPP in RBCs, which was reflected in lower activity of GR and GP, 1.9 times and 2.5 times, respectively, in the main group (Table 2). Moreover, changes in the oxidative status of RBCs of CMV-seropositive pregnant women were characterized by increased (4-fold) cytophotometric indicators of the histochemical reaction products to fatty acids peroxide (Fig.4, Table 3). Subsequent morphological analysis of blood smears revealed structural changes in RBCs, which manifested in increased specific density of the erythrocyte membranes determined at a resolution of 60 pixels (Fig.5). These changes were consistent with degenerative forms, the number of which increased by 3.3 times (Table 3). Automatic calculation of RBC deformability revealed its reduction by 2.25 times (Table. 3), which also indicated the alterations in the structural and functional properties, including those associated with PPP oxygen metabolism.
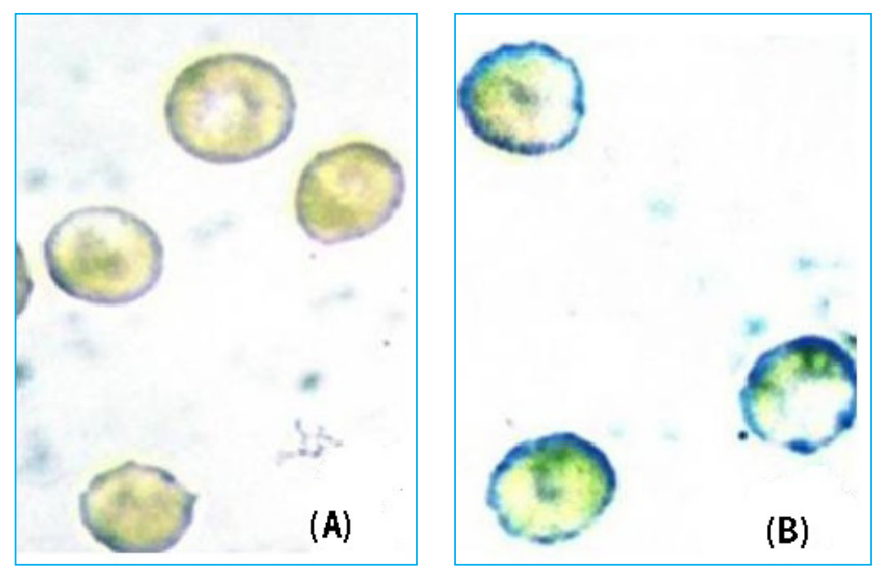

Fig. 2. RBCs of a pregnant woman with CMVI exacerbation at 28 weeks of gestation. Histochemical reaction to reduced NADP according to Lloyd. (A) - main group, $(B)$ - control group. Magnification: $15 \times 100$.
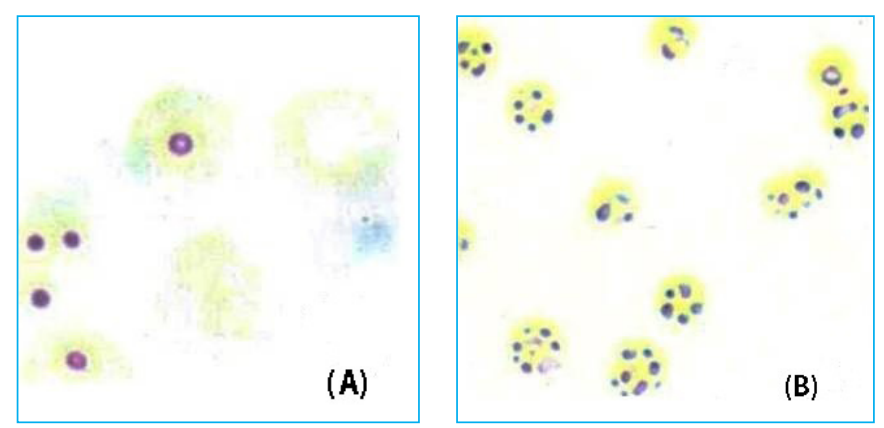

Fig. 3. RBCs of a pregnant woman with CMVI exacerbation at 28 weeks of gestation. Histochemical reaction to reduced glutatione according to MT Lucenko and IA Andriyevskaya. (A) - main group, (B) - control group. Magnification: 10x90.
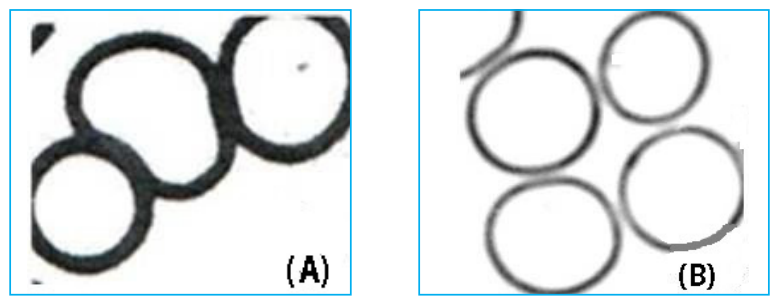

Fig. 4. RBCs of a pregnant woman with CMVI exacerbation at 28 weeks of gestation. Histochemical reaction to fatty acid peroxides according to Winkler-Schulze. (A) - main group, (B) - control group. Magnification: $15 \times 90$. 
Table 2.

Parameters of the functional activity of PPP and GS in RBCs during CMVI exacerbation at 28 to 30 weeks of gestation

\begin{tabular}{|l|c|c|c|}
\hline \multicolumn{1}{|c|}{ Parameters } & Main group & Control group & P value \\
\hline G6PD, pixel $/ \mu \mathrm{m}^{2}$ & $23.0 \pm 0.40$ & $49.75 \pm 0.95$ & $<0.0001$ \\
\hline Reduced NADP, pixel $/ \mu \mathrm{m}^{2}$ & $14.5 \pm 0.25$ & $45.5 \pm 0.8$ & $<0.0001$ \\
\hline Reduced glutatione, $\mathrm{pixel} / \mu \mathrm{m}^{2}$ & $17.6 \pm 1.2$ & $58.6 \pm 0.8$ & $<0.0001$ \\
\hline $\mathrm{GR}, \mathrm{U} / \mathrm{gHb}$ & $4.48 \pm 0.22$ & $8.36 \pm 0.13$ & $<0.0001$ \\
\hline $\mathrm{GP}, \mathrm{U} / \mathrm{gHb}$ & $5.81 \pm 0.13$ & $14.66 \pm 0.36$ & $<0.0001$ \\
\hline
\end{tabular}

Table 3.

Parameters of morphostructure and oxygen metabolism in RBCs during CMVI exacerbation at 28 to 30 weeks of gestation

\begin{tabular}{|l|c|c|c|}
\hline \multicolumn{1}{|c|}{ Parameters } & Main group & Control group & P value \\
\hline Fatty acid peroxides, pixel $/ \mu \mathrm{m}^{2}$ & $95.0 \pm 1.9$ & $23.5 \pm 0.85$ & $<0.0001$ \\
\hline Degenerative forms of RBCs, $\%$ & $15.0 \pm 1.2$ & $4.5 \pm 0.07$ & $<0.0001$ \\
\hline RBC deformability, CU & $0.02 \pm 0.0012$ & $0.045 \pm 0.0018$ & $<0.0001$ \\
\hline 2,3-DPG, $\mu \mathrm{mol} / \mathrm{ml}$ & $7.05 \pm 0.05$ & $5.7 \pm 0.09$ & $<0.0001$ \\
\hline Oxyhemoglobin, \% & $89 \pm 0.8$ & $98 \pm 1.0$ & $<0.0001$ \\
\hline
\end{tabular}
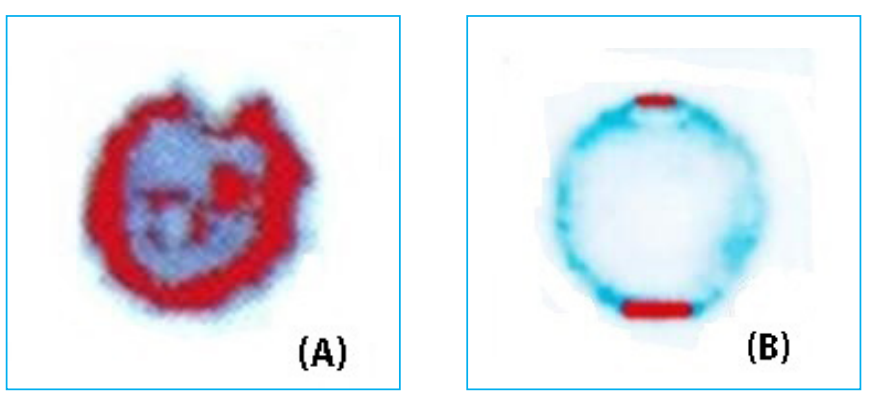

Fig. 5. RBCs of a pregnant woman with CMVI exacerbation at 28 weeks of gestation. Increased specific density of the erythrocyte membranes determined at a resolution of 60 pixels to the BioVision Pixera program. (A) - main group, $(B)-$ control group. Magnification: 10x90.

\section{Secondary endpoints}

The amount of oxyhemoglobin in the main group was 1.1 times less $(\mathrm{P}<0.01)$, whereas the content of 2,3-DPG was 1.24 times more $(\mathrm{P}<0.001)$ than in the control group. Decreasing oxygen metabolism in RBCs during CMVI exacerbation at 28 to 30 weeks of gestation is a result of disturbances in enzyme activity of PPP and GS. In turn, the initiation of oxidation processes contributed to the appearance in the bloodstream of a large number of degenerative forms of RBCs with low capacity for oxygenation, creating the risk of developing hemic hypoxia.

\section{Discussion}

In summary, the present study analyzed the metabolic activity of the enzymes of PPP and GS, and the reduction state of NADP and glutathione in RBCs of women with CMVI exacerbation at 28 to 30 weeks of gestation. We identified the role of CMVI in the development of disorders in functioning and structural equivalence, in both the disulfide reductase system and the oxidative systems of PPP, which determine the processes of erythrocyte oxygenation and the development of hemic hypoxia during pregnancy.

The study of the functional activity of the initial stage of the PPP during CMVI exacerbation at 28 to 30 weeks of gestation was chosen because of known significant changes in the metabolism of RBCs, defined by destabilization and decline in the active state of G6P and reduced NADP that disrupt the thiol-disulfide activity of glutathione..$^{[9-12]}$

The depletion of the pool of reduced glutathione in RBCs causes an energy crisis. ${ }^{[13]}$ The result of this is enhanced proteolytic processes and the processes of lipid peroxidation, increased membrane microviscosity, and reduced deformation properties of membranes, all of which cause destruction of membranes and appearance in the bloodstream of a large number of degenerative forms with altered oxygen metabolism. It should also be noted that formed deficiency of G6PD and GR activity in RBCs may influence the enzymatic transfer and incorporation of iron into heme and thereby may disrupt hemoglobin-forming processes. On the other hand, the accumulation of reactive oxygen species in a form of hydroperoxides leads to an increased oxidation of the globin part that with high 2,3-DPG concentration disrupt oxygenation process and the formation of oxygenated form of hemoglobin, creating a risk of anemia and hemic hypoxia during CMVI exacerbation.

\section{Conclusion}

CMVI exacerbation at 28 to 30 weeks of gestation causes the inhibition of the metabolic activity of the enzymes of PPP and GS, an initiation of membrane destruction oxidative processes that enhance $\mathrm{RBC}$ deformability and decrease oxygen metabolism, all of which create the risk of anemia and hemic hypoxia in pregnant women.

\section{Competing interests}

The authors declare that they have no competing interests.

\section{References}

1. Artemchik TA, Germanenko IG, Kleckij SK. Pathomorphological study of placenta in cytomegalovirus infection. Med Zhurnal. 2012;(3):10-3. [Article in Russian]. 2. Bharara R, Singh S, Pattnaik P, Chitnis C, Sharma A. Structural analogs of sialic acid interfere with the binding of erythrocyte binding antigen-175 to glycophorin A, an interaction crucial for erythrocyte invasion by Plasmodium falciparum. Mol Biochem Parasitol. 2004;138:123-9.

3. Cowman AF, Crabb BS. Invasion of red blood cells by malaria parasites. Cell. 2006;124(4):755-66.

4. Vorob'ev AI. Manual on Hematology. M.: N'yudiamed; 2007, 1274 p. [in Russian].

5. Lucenko MT, Andrievskaya IA. Patent of the Russian Federation, "A method for the determination of glutathione in 
erythrocytes of peripheral blood”. RU № 2526832; register. of 27.08.2014; Bull. № 24. [in Russian].

6. Lucenko MT, Andrievskaya IA. Patent of the Russian Federation, "A method for evaluating the inducing effect of cytomegalovirus infection in the third trimester of gestation on deforming properties of peripheral blood erythrocytes". RU №. 2546532 register. of 10.04.2015; Bull. № 10. [in Russian]. 7. Pokrovskij AA. Biochemical methods of research. M.: Meditsina; 1969, 337 pp. [in Russian].

8. Luganova IS, Blinova MN. Determination of 2,3-phosphoglyceric acid by non-enzymatic method and the content of 2,3-phosphoglycerate and ATP in erythrocytes of patients with chronic lymphocytic leukemia.. Laboratornoe delo. 1975;(6):625-54. [Article in Russian].

9. Salvador A, Savageau MA. Quantitative evolutionary design of glucose-6-phosphote dehydrogenase expression in human erythrocytes. Proc Natl Acad Sci USA. 2003;100(24):14463-8.
10. Vulliam TI, Luzzatto L. Glucose-6-phosphote dehydrogenase deficiency and related disorders. In: Handin RI, Lux SE, Stossel TP (editors). Blood: Principles and practice of hematology. Philadelphia: Lippincott Williams and Wilkins; 2003:1921-50.

11. Kotaka M, Gover S, Vandeputte-Rutten L, Au SW, Lam VM, Adams MJ. Structural studies of glucose-6-phosphote and NADP+ binding to human glucose-6-phosphote dehydrogenase. Acta Crystallogr D Biol Crystallogr. 2005;61(Pt 5):495-504.

12. Cappellini M, Fiorelli G. Glucose-6-phosphate dehydrogenase deficiency. Lancet. 2008; 371(9606):64-74. doi: 10.1016/S0140-6736(08)60073-2.

13. Tang HY, Ho HY, Wu PR, Chen SH, Kuypers FA, Cheng ML, Chiu DT. Inability to maintain GSH pool in G6PD-deficient red cells causes futile AMPK activation and irreversible metabolic disturbance. Antioxid Redox Signal. 2015;22(9):744-59. doi: 10.1089/ars.2014.6142. 